\title{
Whistleblowing für das Weltklima
}

Besprechung des Urteils des Bezirksgerichts Lausanne (Tribunal de Police) PE19.000742/PCL/IIb vom 13. Januar 2020

Das Weltklima ist bedroht und die Politik bleibt untätig. KlimaaktivistInnen sind deshalb dazu übergegangen, mit spektakulären Protestaktionen auf die Folgen des Klimawandels aufmerksam zu machen, etwa indem sie am 22. November 2018 in einer Lausanner Credit-SuisseFiliale Tennis gespielt haben. Damit sollten die klimaschädlichen Investitionen der Bank publik gemacht werden. Die Autoren zeigen auf, dass ein solcher Hausfriedensbruch zwar nicht über einen «Klima-Notstand» gerechtfertigt, dieses Whistleblowing für das Weltklima aber dennoch entschuldigt werden kann.

I. Verfahren

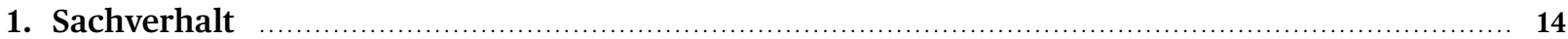

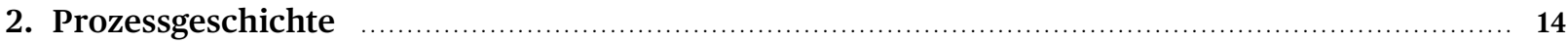

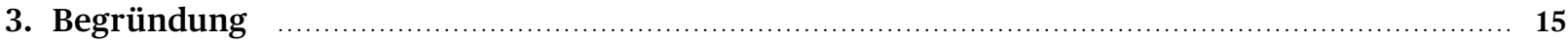

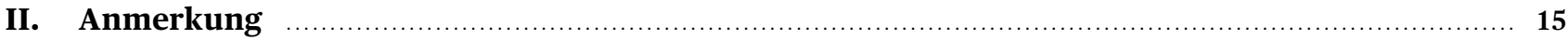

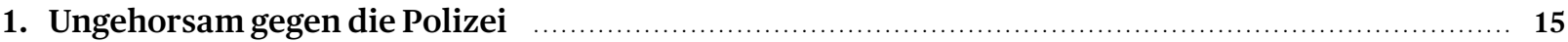

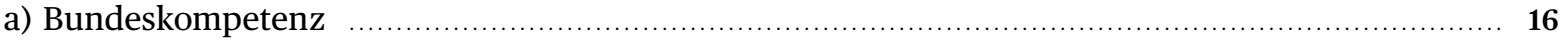

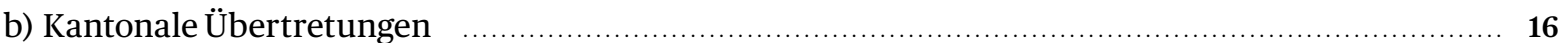

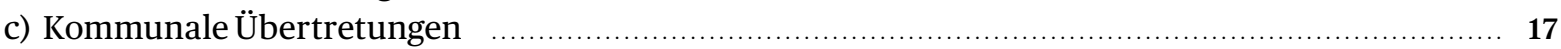

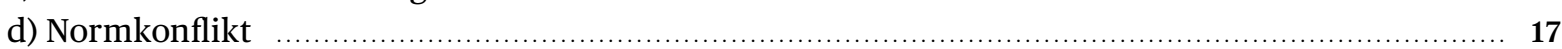

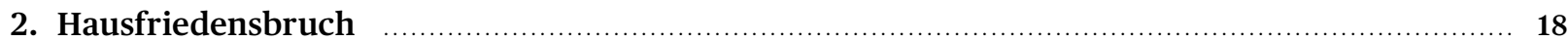

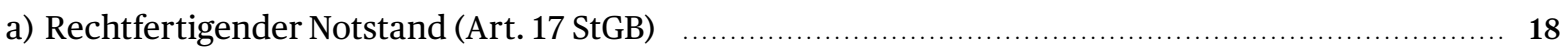

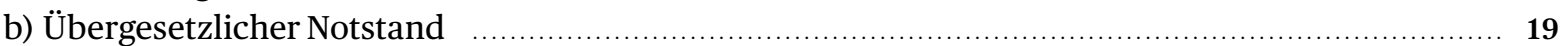

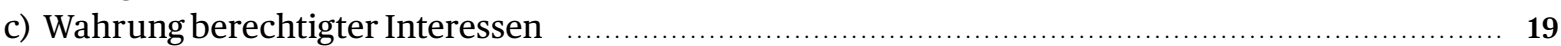

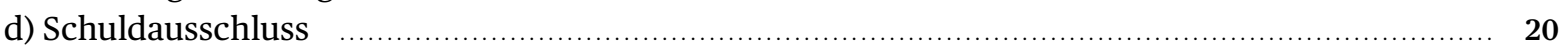

Zitiervorschlag:

MARC THOMMEN / JASCHA MATTMANN, Whistleblowing für das Weltklima, sui generis 2021, S. 13

Prof. Dr. iur. Marc Thommen, Ordinarius für Strafrecht und Strafprozessrecht, UniversitätZürich (marc.thommen@rwi.uzh.ch); Jascha Mattmann, MLaw, wissenschaftlicher Mitarbeiter am Lehrstuhl von Prof. Marc Thommen, Universität Zürich (jaschamatt-

mann@gmail.com). Die Autoren danken Franziska Rader, MLaw, für die sorgfältige Überarbeitung des Manuskripts.

URL: sui-generis.ch/163

DOI: https://doi.org/10.21257/sg.163

Dieses Werk ist lizenziert unter einer Creative Commons Namensnennung - Weitergabe unter gleichen Bedingungen 4.0 International Lizenz. 


\section{Verfahren}

\section{Sachverhalt}

1 Credit Suisse hat zwischen 2016 und 2018 insgesamt 57,4 Milliarden Dollar in fossile Brennstoffe investiert, 1 die für den Grossteil der globalen Treibhausgasemissionen verantwortlich sind. Im Dezember 2019 - und somit nach den hier zu besprechenden Ereignissen - spielte sie eine wichtige Rolle beim Börsengang von Saudi Aramco, dem weltweit wichtigsten Ölexporteur. ${ }^{2}$ Am 22. November 2018 kurz nach 13.00 Uhr betraten knapp 30 als TennisspielerInnen verkleidete Personen die Eingangshalle der Credit-Suisse-Filiale an der Rue du Lion d'Or 5-7 in Lausanne, um dort pantomimisch eine Partie Tennis zu spielen. Sie entrollten ein Transparent mit der Aufschrift: «Crédit Suisse détruit le climat. Roger, tu cautionnes ça? \#SiRogersavait» ${ }^{3}$. Es ging den AktivistInnen darum, auf den Klimawandel im Allgemeinen aufmerksam zu machen und gegen die Investitionen der Credit Suisse in fossile Energien zu demonstrieren. Der Tennisspieler Roger Federer, der seit 2009 von der Credit Suisse gesponsert wird, ${ }^{4}$ wurde dafür kritisiert, dass er sich am Werbeauftritt der Bank beteilige. Im Übrigen verhielten sich die AktivistInnen friedlich und hinderten die Kunden der Bank nicht am Vorbeigehen. Die Aktion war nicht bewilligt. Der Verantwortliche der Filiale forderte die AktivistInnen zunächst vergeblich auf, die Bank zu verlassen, danach verständigte er die Polizei. Diese ersuchte die AktivistInnen, die Bank innert 15 Minuten zu verlassen, was einige von ihnen taten. Als die restlichen AktivistInnen auch dieser Aufforderung nicht nachkamen, lösten die Polizisten die Aktion auf, indem sie diese raustrugen. Um 14.20 Uhr war alles wieder normal in der Filiale. ${ }^{5}$

\section{Prozessgeschichte}

2 Die Credit Suisse (Suisse) SA hat am 28. Dezember 2018 Strafantrag gestellt. Am 25. April 2019 hat die Staatsanwaltschaft des Bezirks Lausanne gegen zwölf der AktivistInnen Strafbefehle erlassen wegen Hausfriedensbruchs (Art.186 StGB) und wegen Verletzung der Arti-

1 BankTrack, Banking on Climate Change-Fossil Fuel Finance Report Card 2019.

2 Handelszeitung vom 12. September 2019 (Die Credit Suisse unterstützt beim Börsengang von Aramco).

3 «Credit Suisse zerstört das Klima. Roger, billigst Du das? \#WennRoger wüsste» [eigene Übersetzung]; vgl. dazu Bild in der NZZ vom 13.Januar 2020 (Ein historisches Urteil: Klima-Aktivisten siegen gegen die Credit Suisse mit 6:0).

4 Dieses Sponsoring wird im Urteilssachverhalt nicht erwähnt, vgl. dazu 20 Minuten vom 16. November 2009 (Credit Suisse wird Sponsor von Roger Federer).

5 Urteil des Bezirksgerichts Lausanne (Tribunal de Police) PE19.000742/PLC/IIb vom 13. Januar 2020, Ziff. 2 «Les faits», S. 41. kel 29 (Hinderung einer Amtshandlung) ${ }^{6}$ und 41 (unbewilligte Demonstration) des Règlement général de police de la Commune de Lausanne (RGP) vom 27. November 2001. ${ }^{7}$ Sie wurden darin zu Geldstrafen von 30 Tagessätzen à 30 Franken bedingt vollziehbar bei einer Probezeit von zwei Jahren und Bussen von 400 bis 600 Franken verurteilt. ${ }^{8}$

Die AktivistInnen haben gegen die Strafbefehle Einspra- 3 che erhoben. Am 7. und 8. Januar 2020 kam es zur Hauptverhandlung vor dem Bezirksgericht in Lausanne. Unter dem Vorsitz des Bezirksgerichtspräsidenten Philippe Colelough (FDP) wurden Prof. Dr. Sonia I. Seneviratne vom Center for Climate Systems Modelling der ETH Zürich, Prof. Dr. Jacques Dubochet, emeritierter Professor für Biophysik der Universität Lausanne und Nobelpreisträger für Chemie (2017) sowie der Finanzanalyst Jérémy Désir als Zeugen sowie sämtliche Angeklagten angehört. Insgesamt 13 StrafverteidigerInnen haben in der Folge plädiert und Freisprüche für alle Angeklagten verlangt. Das Urteil wurde am 13.Januar 2020 mündlich verkündet. Alle Angeklagten wurden freigesprochen und die Kosten auf die Staatskasse genommen. Der Generalprokurator des Kantons Waadt, Eric Cottier, hat gegen das Urteil Berufung eingelegt. ${ }^{9}$ Mit Urteil vom 22. September 2020 hat das Waadtländer Kantonsgericht unter dem Vorsitz von Christophe Maillard (SP) die AktivistInnen im Sinne der Staatsanwaltschaft verurteilt. ${ }^{10}$ Dabei erhielten zehn der zwölf AktivistInnen eine Geldstrafe von 20 Tagessätzen à 20 Franken und eine Busse von 150 Franken. Die restlichen zwei AktivistInnen, die sich der Polizei nicht widersetzten, wurden mit einer Geldstrafe von 10 Tagessätzen à 20 Franken und einer Busse von 100 Franken bestraft. ${ }^{11}$ Die AktivistInnen haben angekündigt, ihre Verurteilung an das Bundesgericht und falls nötig bis nach Strassburg weiterzuziehen. ${ }^{12}$

6 Cum grano salis wurden nur zehn der zwölf AktivistInnen nach Art. 29 RGP verurteilt (Urteil des Bezirksgerichts Lausanne [Tribunal de Police] PE19.000742/PLC/IIb vom 13.Januar 2020, S. 47), weil zwei von Ihnen der Aufforderung der Polizei Folge leisteten und die Bank verliessen.

7 Règlement général de police de la Commune de Lausanne (RGP) vom 27. November 2001.

8 24heures vom 14.Januar 2020 (Activistes du climat: le Procureur va faire appel).

9 24heures vom 14.Januar 2020 (Activistes du climat: le Procureur va faire appel).

10 Urteil des Kantonsgerichts Waadt n ${ }^{\circ} 371$ PE19.000742/PCL vom 22. September 2020, Dispositif II; krit. MARION CHAUTARD, La condamnation en appel des activistes du climat à Lausanne, LawInside vom 7 . November 2020.

11 Urteil des Kantonsgerichts Waadt n 371 PE19.000742/PCL vom 22. September 2020, Dispositif II.

12 ANTONIO FUMAGALLI, Eine Überraschung bleibt aus: Die Klimaaktivisten, die in einer CS-Filiale Tennis gespielt haben, werden in zweiter Instanz verurteilt, NZZ vom 24. September 2020. 

mediales Echo zur Folge. Er wurde u.a. von der BBC ${ }^{13}$ und dem Spiegel ${ }^{14}$ besprochen. Schon vor der Urteilsverkündung war das von den AktivistInnen kreierte Hashtag \#rogerwakeupnow zeitweise auf Platz 1 in der Schweiz, und es wurde von Greta Thunberg retweetet. Roger Federer hat zur Aktion Stellung genommen. ${ }^{15} \mathrm{Un}$ terdessen hat auch die Genfer Strafjustiz einen Klimaaktivisten zweitinstanzlich freigesprochen, der einen roten Handabdruck auf die Fassade eines Credit-SuisseGebäudes angebracht hatte. ${ }^{16}$

\section{Begründung}

5 Das Bezirksgericht hält den Tatbestand des Hausfriedensbruchs (Art.186 StGB) für erfüllt, indem sich die AktivistInnen geweigert haben, die Eingangshalle der Credit Suisse zu verlassen. Ferner seien Art. 29 und 41 des Lausanner Polizeireglements verletzt worden, indem der polizeiliche Räumungsbefehl missachtet wurde. Beide Tatbestände seien jedoch durch Notstand nach Art. 17 StGB gerechtfertigt.

6 Der Notstand ziele zwar grundsätzlich auf den Schutz von Individualrechtsgütern, ausnahmsweise aber auch auf kollektive Interessen, «si un bien juridique personnel est également en jeu». ${ }^{17}$ Konkret habe das Handeln der Angeklagten abgezielt auf «la préservation du climatet de l'environnement et par ce biais la sauvegarde de leur droit personnel à la santé et à la vie»18. Ferner brauche es eine unmittelbare Gefahr. ${ }^{19}$ Das Bezirksgericht stellt primär auf die Gefahren ab, die vom Klimawandel ausgehen ("conséquences du changement climatique»), namentlich dieglobale Erderwärmung («réchauffement planétaire») ${ }^{20}$ und in zweiter Linie auf die Gefahren, die daraus für die Menschheit erwachsen, wie die «fonte des glaces, la montée des eaux, la désertification, l'acidification des océans et l'augmentation des événements extrêmes.» ${ }^{21}$ Diese Gefahren seien unmittelbar, da die Schweiz auf eine Erwär-

13 BBC vom 12.Januar 2020 (Federer responds to climate change critics over Credit Suisse).

14 BIRTE BREDOW, Wir hoffen, dass er tatsächlich aktiv wird und es nicht nur sagt, Spiegel online vom 17. Januar 2020.

15 Watson vom 11.Januar 2020 (Federer lobt Klimajugend - diese will «mehr als nur leere Worte» vom Superstar).

16 PHILIPPE BACH, Climat: et de deux, leCourrier.ch vom 14. Oktober 2020.

17 Urteil des Bezirksgerichts Lausanne (Tribunal de Police) PE19.000742/PLC/IIb vom 13. Januar 2020, S. 49.

18 Urteil des Bezirksgerichts Lausanne (Tribunal de Police) PE19.000742/PLC/IIb vom 13. Januar 2020, S. 54.

19 Urteil des Bezirksgerichts Lausanne (Tribunal de Police) PE19.000742/PLC/IIb vom 13.Januar 2020, S. $48 \mathrm{f}$.

20 Beide Zitate Urteil des Bezirksgerichts Lausanne (Tribunal de Police) PE19.000742/PLC/IIb vom 13. Januar 2020, S. 50.

21 Urteil des Bezirksgerichts Lausanne (Tribunal de Police) PE19.000742/PLC/IIb vom 13. Januar 2020, S. 51. mung von $3{ }^{\circ} \mathrm{C}$ zusteuere, wenn sie weiterhin ihre Verpflichtungen aus dem Pariser Klimaabkommen nicht einhalte. $^{22}$

Nach allgemeinen Verhältnismässigkeitsgrundsätzen 7 dürfe es nicht möglich sein, die Gefahr anderweitig abzuwenden. Es gelte eine absolute Subsidiarität. Hier erwägt das Bezirksgericht drei Alternativen: Erstens hätte eine Kundgebung auf öffentlichen Grund nicht die gleiche mediale Wirkung entfaltet wie die erfolgte Aktion. Zweitens hätten sich die AktivistInnen bereits erfolglos an die Bank gewandt mit ihren Anliegen. Den politischen Weg zu beschreiten, sei drittens dort verzichtbar, wo wie in casu hochwertige Rechtsgüter unmittelbar bedroht sind und ihr Schutz durch die zuständigen Behörden nicht mehr rechtzeitig erfolgen kann. «En termes plus généraux, le temps politique, lent de par sa nature démocratique, n'est plus compatible avec l'urgence climatique averée». ${ }^{23}$

Schliesslich müsse das gewahrte Gut wertvoller sein als 8 das verletzte. Diese Interessenabwägung zwischen den gewahrten Klima-, Gesundheits- und Lebensinteressen und dem minimal verletzten Hausrecht falle vorliegend «indiscutablement en faveur» der Angeklagten aus. ${ }^{24}$ Subjektiv seien den Tätern die Gefahr, die Notwendigkeit, ein Rechtsgut zu retten, sowie der Rechtfertigungsgrund selbst bewusst gewesen. ${ }^{25}$

\section{Anmerkung}

Nachfolgend ist zuerst auf den Ungehorsam gegenüber 9 der Polizei einzugehen (1). Im Zentrum der Urteilsbesprechung steht die Frage, ob der Klima-Notstand einen Hausfriedensbruch rechtfertigt oder - so unsere These-zumindest entschuldigt (2).

\section{Ungehorsam gegen die Polizei}

Den AktivistInnen wird vorgeworfen, sich dem polizei- 10 lichen Räumungsbefehl widersetzt und, indem sie sich gegenseitig an Armen und Beinen festhielten, die Polizei gezwungen zu haben, die AktivistInnen einzeln aus den Räumlichkeiten zu tragen. Damit hätten sie in doppelter Weise (Hinderung der Amtshandlung, Gehorsamsverweigerung) gegen Art. 29 des kommunalen Polizeireglements verstossen. ${ }^{26}$

\footnotetext{
22 Urteil des Bezirksgerichts Lausanne (Tribunal de Police) PE19.000742/PLC/IIb vom 13. Januar 2020, S. 52.

23 Urteil des Bezirksgerichts Lausanne (Tribunal de Police) PE19.000742/PLC/IIb vom 13.Januar 2020, S. 53f.; BGE129 IV 6 E.3.1.

24 Urteil des Bezirksgerichts Lausanne (Tribunal de Police) PE19.000742/PLC/IIb vom 13.Januar 2020, S. 54 f.

25 Urteil des Bezirksgerichts Lausanne (Tribunal de Police) PE19.000742/PLC/IIb vom 13.Januar 2020, S. 49 und 55.

26 Urteil des Bezirksgerichts Lausanne (Tribunal de Police) PE19.000742/PLC/IIb vom 13.Januar 2020, S. 48.
} 

Art. 29 des Lausanner Polizeireglements: «Celui qui, d'une quelconque manière, entrave l'action d'un fonctionnaire, notamment d'un agent de police, ou celui qui refuse deseconformer auxordres d'un agent de police, encourt les peines prévues par la loi sur les contraventions, sans préjudice des sanctions prévues par le Code pénal.»27 einheitlich für die ganze Schweiz. Angesichts dieser bundesrechtlichen Regelung kann man sich fragen, ob die Lausanner Bestimmung bundesrechtskonform ist.

13 Auf den ersten Blick scheint diese Frage geklärt. In BGE 81 IV 163 hat das Bundesgericht entschieden, dass kantonale Bestimmungen, die den Ungehorsam gegenüber der Polizei mit Übertretungsstrafe bedrohen, zulässig seien. ${ }^{28}$ Es hat dies damit begründet, dass das Parlament den «Ungehorsam gegen die Polizei〉 (Art. 339 EStGB/1918) ${ }^{29}$ dem kantonalen Polizeirecht überlassen wollte und ihn deshalb nicht in das Strafgesetzbuch von 1937 übernommen hat. Damit habe der Bundesgesetzgeber klar zum Ausdruck gebracht, «das Feld für kantonales Übertretungsstrafrecht zum Schutze deröffentlichen Gewalt freizugeben». ${ }^{30}$ Die nähere Betrachtung dieses weit über ein halbes Jahrhundert alten Präjudizes zeigt, dass die Gesetzgebungskompetenzen in Bezug auf die strafbaren Handlungen gegen die öffentliche Gewalt zwar zutreffend zwischen Bund (1.a), Kanton (1.b) und Gemeinden (1.c) aufgeteilt werden, das Bundesgericht jedoch die Kollisionsregel von Art. 49 Abs. 1 BV ausser Acht lässt (1.d).

\section{a) Bundeskompetenz}

14 Die Gesetzgebung auf dem Gebiet des Strafrechts ist Sache des Bundes (Art.123 Abs.1 BV). Es handelt sich um eine umfassende, d.h. nicht auf Grundsätze beschränkte, und konkurrierende ${ }^{31}$, d.h. nachträglich derogatorische, Bundeskompetenz. ${ }^{32}$ Der Bund hat von dieser

27 «Wer, auf beliebige Weise, die Handlung eines Beamten, namentlich eines Polizeibeamten, hindert, oder wer sich weigert, Befehle eines Polizeibeamten zu befolgen, wird nach dem Gesetz über die Übertretungen bestraft; Sanktionen nach dem Strafgesetzbuch bleiben vorbehalten» [eigene Übersetzung].

28 BGE 81IV163 (Regeste).

29 Entwurf Schweizerisches Strafgesetzbuch, (BBl 1918IV), S. 205; da zu die Botschaft des Bundesrates an die Bundesversammlung zu einem Gesetzesentwurf enthaltend das schweizerische Strafgesetzbuch vom 23.Juli 1918 (BBl 1918IV), S. 74.

30 BGE 81 IV163 E. 3; vgl. Sitzung des Nationalrats vom 26. September 1929, StenBull 1929 N 616; übernommen in der Sitzung des Ständerats vom 10. Dezember 1931, StenBull 1931S674ff.; MARIANNE JOHANNA HILF, in: Niggli/Wiprächtiger (Hrsg.), Basler Kommentar, Strafrecht, 4. Aufl., Basel 2019, Art.335 N11 (BSK StGB II-BEARBEITERIN).

31 Zum Begriff GIOVIANNI BIAGGINI, in: Biaggini (Hrsg.), Orell Füssli Kommentar, BV, 2. Aufl., Zürich 2017, Vorbemerkungen zu BV 42-135 N11 (OFK BV-BIAGGINI).

32 OFK BV-BIAGGINI, Art. 123 N2.
Kompetenz Gebrauch gemacht durch den Erlass des Strafgesetzbuchs, «dasals Kernstrafrecht Verstössegegen Grundregeln eines friedlichen Zusammenlebens sanktioniert»33. Damit wurde das kantonale Kernstrafrecht grundsätzlich vollständig derogiert. ${ }^{34}$

\section{b) Kantonale Übertretungen}

Die Bundeskompetenz nach Art.123 Abs.1 BV umfasst 15 jedoch nicht das gesamte materielle Strafrecht. Art. 335 Abs.1 StGB statuiert einen echten Vorbehalt zugunsten des kantonalen Übertretungsstrafrechts. ${ }^{35}$ Der Vorbehalt von Art. 335 StGB steht aber seinerseits unter einem Vorbehalt. Kantonales Übertretungsstrafrecht ist nur zulässig, soweit «es nicht Gegenstand der Bundesgesetzgebung ist» (Abs. 1). Der Gegenstand der Bundesgesetzgebung ist durch Auslegung zu ermitteln. ${ }^{36}$

Nach ständiger Rechtsprechung bleibt für kantonales 16 Übertretungsstrafrecht kein Raum, «[s]oweit das Schweizerische Strafgesetzbuch die Angriffe auf ein Rechtsgut durchein geschlossenes System von Normen abschliessend regelt.»37 Hingegen bleibt Raum, wenn der Gesetzgeber nur einen Teil der Tatbestände aus einem bestimmten Gebiet unter Strafe stellt, «um den von Kanton zu Kanton wechselnden Ansichten über die Strafwürdigkeit einer Handlung Rechnung zu tragen»38. Von Letzterem ist der Gesetzgeber bei der Streichung des Ungehorsams gemäss Art. 339 aus dem Entwurf von 1918 ausgegangen: "Das sind Delikte, die bis jetzt im Übertretungsrecht aufgenommen waren, die wir aber auszuschalten wünschen, da sie doch geringfügiger Natur sind und in den kantonalen Polizeigesetzen viel besser behandeltwerden können.»39 Das Bundesgericht hat diese Sichtweise übernommen. Von den sieben ursprünglich im Entwurf vorgesehenen Übertretungen gegen die Staatsgewalt (Art. 337-343 EStGB/1918) habe das Parlament nur drei ins Strafgesetzbuch aufgenommen. Die eidgenössische Ordnung der Straftaten gegen die öffentliche Gewalt (Art. 285ff. StGB) sei deshalb als nicht abschliessend einzustufen und der kantonale Regelungsspielraum zum Ungehorsam gegen die Polizei zu anerkennen. ${ }^{40}$

33 Botschaft über eine neue Bundesverfassung vom 20. November 1996 (BBl1997 I1ff.), S. 340.

34 Zur «Aufhebung des kantonalen Rechts» vgl. Art. 400 Abs.1 StGB (in der bis zum 31. Dezember 2006 geltenden Version); BGE 115 Ia 234 E.12.b; e contrario OFK BV-BIAGGINI, Vorbemerkungen zu BV 42-135 N11.

35 BSK StGB II-HILF, Art. 335 N 7.

36 BGE115 Ia 234 E.12.b.

37 BGE138IV13 E.3.3.1; schon Botschaft über eine neue Bundesverfassung vom 20. November 1996 (BBl1997 I1ff.), S. 340.

38 BGE117 Ia 472 E. 2.b.

39 Votum Berichterstatter NR Seiler in der Sitzung des Nationalrats vom 26. September 1929, StenBull 1929 N616; s.a. Votum NR Logoz, StenBull 1929 N627; ferner Votum NR Seiler in der Sitzung vom 3. März 1930, StenBull 1930 N 46.

40 BGE 81IV163 E.3. 
17 In Bezug auf den 15. Titel des Strafgesetzbuchs ist die Argumentation des Bundesgerichts stringent. Nur einzelne Tatbestände aus dem Gebiet der «Strafbaren Handlungen gegen die öffentliche Gewalt» wurden von Bundesrechts wegen unter Strafe gestellt. Damit bleibt Raum für kantonale Übertretungen.

\section{c) Kommunale Übertretungen}

18 Nach dem Gesagten steht fest, dass die Kantone grundsätzlich die Kompetenz haben, Widerspenstigkeiten gegen die öffentliche Gewalt unter Übertretungsstrafe zu stellen, soweit dies der Bund nicht getan hat. Die Kantone können sodann im Rahmen ihrer Kompetenzen Befugnisse an die Gemeinden delegieren. ${ }^{41}$ Dem Lausanner Polizeireglement lässt sich nicht entnehmen, auf welche Bestimmung der Waadtländer Kantonsverfassung es sich stützt. Grundlage ist wohl Art. 44 Abs. 2 der Constitution du Canton de Vaud du 14 avril 2003 (Sécurité et police): «L'État et les communes assurent l'ordre public ainsi que la sécurité des personnes et des biens.» Auch die Gemeinde Lausanne war somit grundsätzlich befugt, bundesrechtlich nicht geregelte Handlungen gegen die öffentliche Gewalt unter Strafe zu stellen. ${ }^{42}$

\section{d) Normkonflikt}

19 Es wurde gezeigt, dass die Kantone und Gemeinden mangels einer abschliessenden bundesrechtlichen Regelung der strafbaren Handlungen gegen den Staat die Kompetenz haben, im verbleibenden Bereich Übertretungsstrafrecht zu erlassen. Diese parallele Regelungskompetenz kann jedoch dazu führen, dass «inhaltlich gegensätzliche Normen aufdenselben Sachverhalt Anwendung zu finden beanspruchen». Man spricht dann von «Normkonflikten».43 Hier gilt, dass Bundesrecht entgegenstehendem kantonalem Recht vorgeht (Art. 49 Abs. 1 BV). Nach bundesgerichtlicher Rechtsprechung dürfen die Kantone nur solche Vorschriften erlassen, die nicht gegen Sinn und Geist des Bundesrechts verstossen und dessen Zweck nicht beeinträchtigen oder vereiteln. ${ }^{44}$ Ein solcher Normkonflikt zwischen Bundes- und kantonalem resp. kommunalem Recht besteht beim Ungehorsam gegen die Polizei.

20 Nach Art. 29 des Lausanner Polizeireglements wird bestraft, wer die Handlung eines Polizeibeamten hindert, oder wer sich weigert, Befehle eines Polizeibeamten zu befolgen. Nach Art. 286 Abs. 1 StGB macht sich der Hinderung einer Amtshandlung strafbar, wer Beamte an einer Handlung hindert, die innerhalb ihrer Amtsbefug-

\footnotetext{
41 BSK StGB II-HILF, Art. 335 N 5.

42 Zum Vollzug siehe Art.18 RGP und Art. 8 Loi sur les contraventions du Canton de Vaud du 19 mai 2009 (LContr; RSV 312.11).

43 Beide Zitate von OFK BV-BIAGGINI, Art. 49 N 7.

44 BGE138I468 E.2.3.1.
}

nisse liegt. Nach ständiger Rechtsprechung fällt unter Art. 286 StGB, wer einen Beamten durch eine aktive Handlung von einer Amtshandlung abhält, 45 etwa indem er eine amtliche Versteigerung stört ${ }^{46}$ oder vor einer polizeilichen Ausweiskontrolle flüchtet. ${ }^{47}$ Ebenso unbestritten ist in der Rechtsprechung umgekehrt, dass das blosse Nichtbefolgen amtlicher Befehle keine Straftat nach Art. 286 StGB darstellt. ${ }^{48}$ Protestaktionen können straflosen «passiven Widerstand» ${ }^{49}$ darstellen oder tatbestandsmässige aktive Widersetzungen umfassen. Die Rechtsprechung tendiert (wenig überzeugend) dazu, selbst vollkommen passive Proteste wie eine mündliche Weigerung, eine Türe aufzuschliessen ${ }^{50}$ oder die Weigerung einer Gruppe Studierender, eine Fakultätsversammlung zu verlassen, als strafbare "Aktionen» zu qualifizieren. ${ }^{51}$ Gleich wurde für die Bildung einer Menschentraube entschieden. ${ }^{52}$ Diese strenge Rechtsprechung dürfte mit der Versammlungsfreiheit nach Art. 11 EMRK nicht in Einklang zu bringen sein. ${ }^{53}$

Übertragen auf den vorliegenden Fall ergeben sich somit 21 folgende Kollisionen: Die Gehorsamsverweigerung der AktivistInnen ist nach Art.29 des Polizeireglements strafbar, nach Art. 286 StGB hingegen straflos. Der Sitz-

\section{BGE133 IV 97 E.4.2.}

46 Beispiel von Stefan Trechsel / Hans Vest, in: Trechsel/Pieth (Hrsg.), Praxis Kommentar, Strafrecht, 3. Aufl., Zürich 2017, Art. 286 N 3 (zit. PK StGB-BEARBEITERIN).

47 BGE124IV 127 (Regeste); eine mit Blick auf nemo tenetur allerdings sehr fragwürdige Rechtsprechung, dazu MARC THOMMEN / MICHA NYDEGGER, Strafbares Streben nach Freiheit?, sui generis 2018, S. $255, \mathrm{~N} 38 \mathrm{ff}$.

48 BGE 81IV163 E. 2; BGE124IV127 E. 3.a; BGE127IV115 E. 2 und BGE133 IV97 E. 4.2.; eingehend ROBERT SCHNETZER, Die Abgren zung der Hinderung einer Amtshandlung gemäss Art. 286 StGB vom blossen Ungehorsam, Diss. Basel 1979, S. $71 \mathrm{ff}$.

49 GÜNTER STRATENWERTH / FELIX BOMMER, Schweizerisches Strafrecht, Besonderer Teil II: Straftaten gegen Gemeininteressen, 7. Auflage, Bern 2013, \$52 N11, setzen den passiven Widerstand z.R. mit blossem (und damit straflosem) Ungehorsam gleich; zur nach Art. 286 StGB straflosen «passiven Renitenz» vgl. BGE 81 IV 325. In Deutschland sind Sitzblockaden gemäss dem Entscheid des Bundesverfassungsgerichts BVerfGE 92, 1 keine gewaltsamen Nötigungen nach $\$ 240 \mathrm{Abs} .1 \mathrm{StGB} / \mathrm{D}$.

50 Urteil des Bundesgerichts 6B_89/2019 vom 17. Mai 2019 E.1.4.

51 BGE107IV 113 E. 4.d; i.d.S. auch Urteil des Kantonsgerichts Waadt $\mathrm{n}^{\circ} 371$ PE19.000742/PCL vom 22. September 2020 E. 5.1.2 und 5.2.3 und VERONICA BOETON ENGEL / AUDE BICHOVSKY, in: Jeanneret/ Kuhn/Perrier Depeursinge (Hrsg.), Commentaire Romand, Code de procédure pénale suisse, 2. Aufl., Basel 2019, Art. 286 N 8.

52 BGE127IV115 E. 2; siehe auch GUIDO JENNY, Die strafrechtliche Rechtsprechung des Bundesgerichts im Jahre 2001 (ohne Entscheide betreffend die internationale Zusammenarbeit in Strafsachen), ZBJV 140/2004, Band127, S. 18.

53 Überzeugend Richterin Aleksandra Fonjallaz in ihrer «Avis minoritaire», Urteil des Kantonsgerichts Waadt nº 371 PE19.000742/PCL vom 22. September 2020, m.H.a. Urteil des EGMR 51346/99 vom 9. April 2002 (Cissé gegen Frankreich), §§ 39 f.; Urteil des EGMR 14237/07 vom 11. Oktober 2018 (Tuskia und weitere gegen Georgien), $\$ \$ 73$ ff. Im letzteren Fall wurde die staatliche Reaktion auf den Protest u.a. deshalb als noch mit der Versammlungsfreiheit nach Art. 11 EMRK vereinbar eingestuft, weil die strafrechtlichen Vorwürfe fallengelassen wurden. 
streik soll gemäss Bezirksgericht Lausanne nach Art. 29 des Polizeireglements strafbar sein, als rein passive Protestaktion fällt er bei konventionskonformer Interpretation indes nicht unter Art. 286 StGB. Für beide Sachverhaltsvarianten ordnet das kommunale Recht somit Strafbarkeit, das Bundesrecht Straflosigkeit an. Art. 29 des Lausanner Polizeireglements behält die Bestrafung nach dem Strafgesetzbuch ausdrücklich vor ("sans préjudice des sanctions prévues par le Code pénal»54). Eine solche Bestrafung kommt vorliegend nicht in Betracht, weil die Handlungen der AktivistInnen nach dem Strafgesetzbuch nicht strafbar sind. Die Gehorsamsverweigerung und der rein passive Sitzstreik der AktivistInnen sind von Bundesrechts wegen nicht tatbestandsmässig. Das Bundesrecht verdrängt in diesem Fall das kantonale Recht. 55 Die Frage der Rechtfertigung stellt sich damit nicht.

\section{Hausfriedensbruch}

Abgesehen von der Legitimation zur Strafantragsstellung56 war der Hausfriedensbruch nicht umstritten. ${ }^{57}$ Kontrovers war vielmehr, ob sich die AktivistInnen dabei auf einen «Klima-Notstand» berufen konnten. Das Bezirksgericht bejahte dies. Nachfolgend wird zunächst dargelegt, weshalb weder die Voraussetzungen eines rechtfertigenden (2.a) noch eines übergesetzlichen Notstands (2.b) vorliegen. Sodann fragen wir, ob die AktivistInnen in der Wahrung berechtigter Interessen gerechtfertigt (2.c) oder zumindest entschuldigt waren (2.d).

\section{a) Rechtfertigender Notstand (Art. 17 StGB)}

23 Wer eine mit Strafe bedrohte Tat begeht, um ein eigenes oder das Rechtsgut einer anderen Person aus einer unmittelbaren, nicht anders abwendbaren Gefahr zu retten, handelt rechtmässig, wenn er dadurch höherwertige Interessen wahrt (Art.17 StGB). Die Voraussetzungen des rechtfertigenden Notstands sind vorliegend nicht erfüllt.

24 Nach dem Wortlaut des Gesetzes muss eine Notlage bestehen für «ein eigenes oder das Rechtsgut einer anderen Person». Als Objekt des Notstands kommen somit nur Individualrechtsgüter der AktivistInnen (Notstand) oder von Dritten (Notstandshilfe) in Frage, 58 nicht hin-

\footnotetext{
54 «Wer, auf beliebige Weise, die Handlung eines Beamten, namentlich eines Polizeibeamten, hindert, oder wer sich weigert, Befehle eines Polizeibeamten zu befolgen, wird nach dem Gesetz über die Übertretungen bestraft; Sanktionen nach dem Strafgesetzbuch bleiben vorbehalten.» [eigene Übersetzung].

55 Zur umstrittenen Rechtsfolge von Normkonflikten schlägt OFK BVBIAGGINI, Art. 49 N 8 überzeugend «die Verdrängung im fraglichen Anwendungsfall (‘Anwendungsvorrang))» vor.

56 Dazu MONIKA SIMMLER/GREGOR HÄNE, Strafantragsberechtigung beim Hausfriedensbruch, AJP 2020, S.1112 ff.

57 Urteil des Bezirksgerichts Lausanne (Tribunal de Police) PE19.000742/PLC/IIb vom 13. Januar 2020, S. 43 ff.

58 So auch ständige Rechtsprechung und h.L.: BGE 94 IV 68 E. 2; BSK StGB-NIGGLI/GÖHLICH, Art. 17 N 5.
}

gegen kollektive Rechtsgüter wie Umwelt- und Klimaschutz ${ }^{59}$. Bereits in Bezug auf die gewahrten Interessen ist das Urteil zweideutig: Es sei den AktivistInnen um die Erhaltung des Klimas und der Umwelt und «damit» («par ce bias») um die Rettung ihrer Gesundheit und ihres Lebens gegangen. ${ }^{60}$ Zumindest letztere sind Individualrechtsgüter der AktivistInnen selbst. Das Bezirksgericht scheint somit von einem eigenen Notstand der AktivistInnen auszugehen.

Der Täter handelt nur rechtmässig, wenn er die Straftat 25 begeht, um das Rechtsgut aus einer Gefahr zu retten (Art. 17 StGB). Weil das Bezirksgericht nicht exakt festlegt, welche Rechtsgüter auf dem Spiel stehen, bleiben auch seine Ausführungen zur Gefahr vage. Primär wird auf die Gefahren abgestellt, die vom Klimawandel ausgehen ("conséquences du changement climatique»), namentlich die globale Erderwärmung («réchauffement planétaire») ${ }^{61}$. Erst in zweiter Linie wird erwähnt, dass daraus auch Gefahren für die Menschheit erwachsen, wie die «fonte des glaces, la montée des eaux, la désertification, l'acidification des océans et l'augmentation des événements extrêmes.»62 Richtigerweise wären die Gefahren, welche etwa von Treibhausgasen für das Klima ausgehen, zu unterscheiden gewesen von den Gefahren, die der Menschheit aufgrund der Klimaerwärmung drohen. Nur Gefahren für Menschen und ihre Individualrechtsgüter sind bei Art. 17 StGB relevant.

Die Gefahr muss unmittelbar sein (Art.17 StGB). Bereits 26 hier gerät das Bezirksgericht auf Abwege. Statt darzulegen, inwiefern sich die AktivistInnen im Moment, in dem sie als TennisspielerInnen verkleidet die Eingangshalle der Bank betraten, sich in unmittelbarer Lebensoder Gesundheitsgefahr befanden, macht es Ausführungen zur Umsetzung des Pariser Klimaabkommens in der Schweiz. Hier zeigt sich, dass es wohl nähergelegen hätte, auf Notstandshilfe abzustellen, zumal es zahlreiche Menschen gibt, die etwa durch klimawandelbedingte Gletscherabbrüche oder Bergstürze unmittelbar in ihrem Leben bedroht sind. ${ }^{63}$

59 Falls diese überhaupt als eigenständige Rechtsgüter in Frage kom men, siehe dazu JÜRG-BEAT ACKERMANN / SAMUEL EGLI, UmweltWirtschaftsstrafrecht. Rechtsgüter-Normkonzepte-Sanktionen, in: Ackermann/Hilf(Hrsg.), Umwelt-Wirtschaftsstrafrecht, 9. Schweizerische Tagung zum Wirtschaftsstrafrecht, Zürich et al. 2017, S.13.

60 Urteil des Bezirksgerichts Lausanne (Tribunal de Police) PE19.000742/PLC/IIb vom 13. Januar 2020, S. 54.

61 Beide Zitate im Urteil des Bezirksgerichts Lausanne (Tribunal de Police) PE19.000742/PLC/IIb vom 13.Januar 2020, S. 50.

62 Urteil des Bezirksgerichts Lausanne (Tribunal de Police) PE19.000742/PLC/IIb vom 13.Januar 2020, S. 51.

63 ANDRÉS PAYER, Klimawandel als strafrechtlicher Notstand, sui generis 2020, N13; ARNAUD NUSSBAUMER, L'acquittement des activis tes du climat à Lausanne, LawInside vom 21. Februar 2020, S. 3. 

standshandlung nicht nur strengen Subsidiaritäts ${ }^{64}$ und Proportionalitätsanforderungen unterliegt, sondern auch geeignet sein muss, die Gefahr abzuwenden. ${ }^{65}$ Spätestens hier scheitert die Rechtfertigung:66 Ein TennisMatch in einer Lausanner Bankfiliale rettet weder die bestenfalls mittelbar bedrohten Leben der AktivistInnen noch die unmittelbar bedrohten Leben von BergbauerInnen und WüstenbewohnerInnen.

\section{b) Übergesetzlicher Notstand}

Das Bundesgericht anerkennt einen «übergesetzlichen» Notstand, den es in seinen Voraussetzungen eng an den gesetzlichen Notstand anlehnt. ${ }^{67}$ Danach können Anliegen ausnahmsweise mit strafbaren Methoden durchgesetzt werden, «wenn eine notstandsähnliche Gefahrenlage gegeben istbzw.wenn hochwertige Rechtsgüter unmittelbar bedroht sind undihrSchutzdurch die zuständigen Behörden nicht mehr rechtzeitig erfolgen kann.» ${ }^{68}$ Der Unterschied zu Art. 17 StGB besteht darin, dass der übergesetzliche Notstand darauf abzielt, Gefahren für Rechtgüter der Allgemeinheit zu beseitigen oder einzudämmen. ${ }^{69}$ Soweit es den AktivistInnen um den Schutz der Umwelt resp. der Menschheit vor den Gefahren des Klimawandels ging, ${ }^{70}$ zielte ihr Handeln auf die Rettung eines kollektiven Rechtsguts. ${ }^{71}$ Doch scheitert die Rechtfertigung auch hier daran, dass die primär auf Publikumswirksamkeit zielende Aktion nicht geeignet war, die vom Klimawandel ausgehenden Gefahren zu beseitigen. Mit der Besetzung einer Bankfiliale lässt sich das Weltklima nicht retten. ${ }^{72}$

64 GÜNTER STRATENWERTH, Strafrecht Allgemeiner Teil I, 4 . Aufl., Bern 2011, §10 N 43.

65 BGE109IV156 E.3; ferner KURT SEELMANN / CHRISTOPHER GETH, Strafrecht Allgemeiner Teil, 6. Aufl., Basel 2016, N166.

66 So auch PAYER (Fn. 63), N17f.; GRACE SCHILD TRAPPE / FELIX SCHÖBI, Not kennt kein Gebot beim Hausfriedensbruch?, Jusletter vom 11. Mai 2020, N17 und 20; a.M. NUSSBAUMER (Fn. 63), S. 6 f.

67 BGE 94 IV 68, Regeste Ziff. 1 sowie E. 2.

68 BGE129IV6 Ziff.3.1.

69 Vgl. ANDRÉS PAYER, Zur Wahrnehmung berechtigter Interessen im Strafrecht, recht 2020, S.190f.; noch enger STRATENWERTH (Fn. 64), \$10 N 58, der eine Bedrohung der «Lebensinteressen des Staats» verlangt.

70 Zum Streit um öko- und/oder anthropozentrische Dimension des Umweltschutzes, vgl. JÜRG-BEAT ACKERMANN / SAMUEL EGLI, UmweltWirtschaftsstrafrecht. Rechtsgüter-Normkonzepte-Sanktionen, in: Ackermann/Hilf(Hrsg.), Umwelt-Wirtschaftsstrafrecht, 9. Schweizerische Tagung zum Wirtschaftsstrafrecht, Zürich 2017, S.16f.

$71 \mathrm{Zu}$ den im Umweltstrafrecht geschützten Rechtsgütern vgl. MARIANNE JOHANNA HILF / HANS VEST, Gutachten «Umweltstrafrecht» im Auftrag des BAFU, Bern 2016, S. $32 \mathrm{ff}$.

72 Immerhin hat die Aktion und die damit verbundene negative Publicity die Credit Suisse zu einem Einlenken bewogen, indem sie etwa die Finanzierung neuer Kohlekraftwerke stoppt (dazu FLoRIAN A. LEHMANN, CS will keine neuen Kohlekraftwerke mehr finanzieren, Tagesanzeiger vom 11. Dezember 2019), und mit LYDIE HUDSON eine CEO für Nachhaltigkeit eingesetzt hat (PETER HODY, Die «grüne» Credit Suisse meint es ernst, finews.ch vom 2. September 2020), was sich zumindest mittelbar positiv auf den Klimawandel auswirken dürfte.

\section{c) Wahrung berechtigter Interessen}

Das Bundesgericht stuft auch die Wahrung berechtigter 29 Interessen ${ }^{73}$ als notstandsähnlichen Rechtfertigungsgrund ein, auf den die Notstandsvoraussetzungen analog anzuwenden sind. ${ }^{74}$ Die Wahrung berechtigter Interessen setze daher voraus, dass die Tat ein zur Erreichung des berechtigten Ziels notwendiges und angemessenes Mittel ist, sie insoweit den einzig möglichen Weg darstellt und offenkundig weniger schwer wiegt als die Interessen, welche der Täter zu wahren sucht. ${ }^{75}$ Der Interessenkonflikt darf gesetzlich nicht bereits geregelt ${ }^{76}$ und legale Mittel müssen ausgeschöpft sein. ${ }^{77}$ Es besteht eine bunte Palette von Anwendungsfällen, ${ }^{78}$ deren gemeinsamer Nenner nicht die Gefahrenabwehr, sondern die Herstellung sozial erwünschter Zustände oder die Ausübung verfassungsmässig garantierter Freiheitsrechte auf Kosten anderer Interessen ist. ${ }^{79}$

Soweit es um die Gefahrenabwehr geht, besteht mit dem 30 Notstand bereits eine gesetzliche Regelung. In Betracht kommt daher das Ziel der AktivistInnen, die Öffentlichkeit auf die Folgen des Klimawandels im Allgemeinen und die klimaschädlichen Investitionen der Credit Suisse im Besonderen hinzuweisen. Dass der Klimaschutz nicht nur sozial erwünscht sondern auch rechtlich geboten ist, ergibt sich nur schon daraus, dass die Schweiz das Pariser Klimaübereinkommen ratifiziert hat. ${ }^{80}$ Implizit gehen diese Ziele auch aus Art. 73 (Nachhaltigkeit), Art. 74 (Umweltschutz) ${ }^{\mathbf{8 1}}$ und Art. 89 (Energiepolitik) ${ }^{82}$ BV hervor. Ferner geniesst das Vorgehen der AktivistInnen grundrechtlichen Schutz. Sie übten damit

73 Terminologisch postuliert PAYER (Fn. 69), S.186 als Oberbegriff die Wahrnehmung berechtigter Interessen, die in der (negativ-defensiven) Wahrung oder der (positiven) Durchsetzung berechtigter Interessen liegen kann.

74 BGE 94IV68E.2.

75 BGE134 IV 216 E. 6.1; BGE127 IV122 E.5.c; BGE 127 IV 166 E. 2.b; BGE126IV 236 E. 4.b.

76 BGE120IV 208 E. 3.

77 STRATENWERTH (Fn. 64), \$10 N61.

78 STRATENWERTH (Fn. 64), §10 N 59.

79 SEELMANN/GETH (Fn.65), N183; siehe auch STRATENWERTH (Fn. 64), §10 N60 ff.; kategorisch ablehnend, da eine allg. Interessenverrechnung die Grenzen strafbaren Verhaltens komplett auflöse, BSK StGB-NIGGLI/GÖHLICH, Vor Art.14 N66ff.; HANS SCHULTZ, Einführung in den allgemeinen Teil des Strafrechts, Bd. I, 4. Aufl., Bern 1982, S.172; beschwichtigend FRANZ RIKLIN, Straffreiheit bei Wahrung berechtigter Interessen, Medialex 2018, S. 24, N 7; DANIEL JOSITSCH / CLAUDIA V. BRUNNER, Whistleblowing als Rechtfertigungsgrund, AJP 2012, S. 485.

80 Klimaübereinkommen von Paris vom 12. Dezember 2015 (SR 0.814.012), in Kraft getreten für die Schweiz am 5. November 2017.

81 Vgl. OFK BV-BIAGgini, Art. 73 N 2 und Art. 74 N4.

82 Botschaft über einen Energieartikel in der Bundesverfassung vom 7. Dezember 1987 (BBl1988 I337 ff.), S. 376. 
ihre Meinungsäusserungs- und Versammlungsfreiheit aus (Art. 16 und 22 BV, Art. 10 und 11 EMRK). ${ }^{83}$ Es ging um eine Art «Whistleblowing für das Weltklima». dung der Klimagefahren zu beziehen, sondern auf die angestrebte Sensibilisierung. Bereits das Tennis-Spiel fand ein grosses Medienecho ${ }^{84}$ und war insofern geeignet, die Aufmerksamkeit der Öffentlichkeit auf die Anliegen der AktivistInnen (Klimawandel, klimaschädliche Investitionen der CS) zu lenken. Paradoxerweise war es vorliegend aber gerade das durch die Begehung einer Straftat provozierte Gerichtsverfahren, welches den AktivistInnen die mediale Weltbühne bereitet hat. Eine legale Demonstration hätte kein solches Echo gefunden. Noch paradoxer ist, dass die Credit Suisse den medienträchtigen Showdown im Gerichtssaal überhaupt erst ermöglicht hat, indem sie von ihrem guten Recht Gebrauch machte, Strafantrag zu stellen.

Bei der Erforderlichkeit ist die Gerichtspraxis streng und verlangt, dass «[i]n einem demokratischen Rechtsstaat (...) politische und ideelle Anliegen grundsätzlich auf politischem Wege bzw. aufdem Rechtsweg zu verfolgen sind. (...) [D] ass die legalen (...) Möglichkeiten ausgeschöpft erscheinen (...) gibt Letzteren kein Recht, ihre Anliegen mit strafbaren Methoden zu verfolgen.» ${ }^{85}$ Das Bezirksgericht hat dargelegt, dass vorangehende legale Interventionen der AktivistInnen erfolglos geblieben sind. ${ }^{86}$ Nach überzeugender Ansicht ist die Erforderlichkeit zu bejahen, wenn «das gravierendste zugleich das aussichtsreichste Mittel» ist. ${ }^{87}$ Vorliegend war die Begehung des Hausfriedensbruch zwar gravierender als alle vorangehenden Manifestationen, aber eben auch um ein Vielfaches wirksamer. Um eine Aufrüttelung der Öffentlichkeit in diesem Umfang zu erreichen, war die Begehung eines Hausfriedensbruchs somit erforderlich.

33 War die Aktion verhältnismässig im engeren Sinne? Aus der Optik des Bezirksgerichts fällt die Interessenabwägung «indiscutablement en faveur des (...) prévenus» aus: Die gewahrten Klimaschutz-, Lebens- und Gesundheitsinteressen überwögen das bloss marginal tangierte Hausrecht der Bank klar. ${ }^{88}$ Wie beim Notstand darge-

83 Zur Bedeutung der Kommunikationsgrundrecht REGINA KIENER / WALTER KÄLIN, Grundrechte, S. 202, und zu den hohen Anforderungen an ihre Einschränkung S. 216.

84 20 Minutes vom 23. November 2018 (On veut informer Roger sur les actes de la banque).

85 BGE129IV6 Ziff.3.1.

86 Urteil des Bezirksgerichts Lausanne (Tribunal de Police) PE19.000742/PLC/IIb vom 13. Januar 2020, S. $52 \mathrm{ff.}$

87 PAYER (Fn.69), S.192.

88 Urteil des Bezirksgerichts Lausanne (Tribunal de Police) PE19.000742/PLC/IIb vom 13. Januar 2020, S. 54 f. legt, können die Klimaschutz- und Lebensinteressen indes nicht direkt zur Abwägung gestellt werden, weil das Tennis-Spiel in der Bank jedenfalls nicht geeignet war, diese Gefahren zu beseitigen. ${ }^{89}$ Bei der Wahrung berechtigter Interessen muss beurteilt werden, ob das Interesse, die Öffentlichkeit wachzurütteln und sie auf die Gefahren des Klimawandels und die klimaschädlichen Investitionen der Credit Suisse hinzuweisen, schwerer wiegt als das Hausrecht der Bank. Ob in dieser Abwägung gegen die AktivistInnen spricht, dass sie das «Interesse der Allgemeinheit an der Ordnungs- und Friedensfunktion» ${ }^{90}$ beeinträchtigt haben, ist zweifelhaft. Jede Straftat bedroht den Rechtsfrieden. Für die AktivistInnen streitet in dieser Abwägung eine Analogie zum Defensivnotstand ${ }^{91}$ : Die Aktion wandte sich nicht wie in BGE134 IV 216 gegen unbeteiligte Dritte, sondern gegen eine juristische Person, die für die angeprangerten Missstände mitverantwortlich ist.

Zusammenfassend spricht Vieles dafür, den AktivistIn- 34 nen zuzubilligen, dass sie berechtigte und überwiegende Interessen gewahrt haben und deshalb zu rechtfertigen sind. Gegen eine Rechtfertigung spricht die rechtspolitische Überlegung, dass man damit nicht nur der Bank das Notwehrrecht abschneidet, sondern auch der Polizei verunmöglicht einzugreifen.92 $\mathrm{Zu}$ prüfen bleibt somit, ob man die AktivistInnen entschuldigen kann.

\section{d) Schuldausschluss}

Andrés Payer lehnt im vorliegenden Fall einen Schuld- 35 ausschluss ab. Er schlägt vor, nach Art. 52 StGB von einer Strafe abzusehen, weil sowohl das objektiv verwirklichte Unrecht als auch das subjektive Verschulden der Täter gering sind. Einen Schuldspruch hält er für sozialpolitisch geboten, um den Regelverstoss zu missbilligen. ${ }^{93}$ Für diese Lösung spricht erstens, dass AktivistInnen, welche sehenden Auges illegale Mittel wählen, um auf ihre Anliegen aufmerksam zu machen, auch der Tadel einer Verurteilung zumutbar ist. Die ganze Aktion war darauf angelegt, sich bei einer Verurteilung als Märtyrer einer klimablinden Justiz inszenieren zu können. Ansonsten hätten sie die Bank ja bei der ersten Aufforde-

89 Zu den Auswirkungen der Protestaktion, welche zur mittelbaren Eindämmung der Klimagefahren beitragen können, vgl. Fn. 72.

90 PAYER (Fn.69), S. 193.

91 PK StGB-TREChSEL/Geth, Art. 17 N 4; GIAN MARTIN, Defensivnotstand unter besonderer Berücksichtigung der «Haustyrannentötung», Diss. Zürich 2010, S. 43 ff.; nicht überzeugend a.A. BSK StGBNIGGLI/GÖHLICH, Art. 17 N 19.

92 Insoweit überzeugend TRAPPE/SCHÖBI (Fn. 66), N11; zur identischen Argumentation (nur Schuldausschluss trotz Wahrung überwiegender Interessen) beim Nötigungsnotstand, JOHANNES WESSELS / WERNER BEULKE, Strafrecht (StrafR) Allgemeiner Teil, 40. Aufl., N443; KRISTIAN KÜHL, Strafrecht. Allgemeiner Teil, 3. Aufl., N127ff. 93 PAYER (Fn. 63), N30 ff. 
rung verlassen können. Zweitens liegt der Vorschlag auf der Linie der bundesgerichtlichen Rechtsprechung, die es der Staatsanwältin bei Vorliegen der Voraussetzungen von Art. 52 StGB zwar erlaubt, das Verfahren einzustellen, das Gericht hingegen verpflichtet, einen Schuldspruch auszufällen.94 Da nicht davon auszugehen ist, dass das Bundesgericht demnächst von dieser Rechtsprechung abweichen wird, muss der Schuldausschluss unabhängig von Art. 52 StGB begründet werden.

Das minimal invasive und subtil humorvolle ${ }^{95}$ Vorgehen der AktivistInnen sowie das schiere Gewicht ihrer Anliegen sprechen vorliegend für einen Freispruch. Ein strafrechtlicher Schuldspruch mit Sanktionen dürfte zudem eine disproportionale Beeinträchtigung der Ver-

94 BGE135IV 27 (Regeste); z.R. sehr kritisch BSK StGB-RIKLIN, Vor Art. 52-55 N18 und GERHARD FIOLKA / CHRISTOF RIEDO, in: Niggli/ Heer/Wiprächtiger (Hrsg.), Basler Kommentar, Schweizerische Strafprozessordnung/Jugendstrafprozessordnung, 2. Aufl., Basel 2014, Art. 8 N105ff.

95 Gleicher Meinung FonJALlAZ, Avis minoritaire (Fn. 10) («La manifestation ... était empreinte de bonhommie et d'humour »). sammlungsfreiheit nach Art.11 EMRK darstellen.96 Will man die AktivistInnen nicht bereits rechtfertigen, so trifft sie zumindest kein Schuldvorwurf. In der Sache liegt eine entschuldigende Wahrung berechtigter Interessen vor. Die vom Bundesgericht gezogene Analogie zum Notstand ${ }^{97}$ legt es nahe, auch bei der Wahrung berechtigter Interessen nicht nur die Rechtfertigung, sondern a maiore ad minus auch den Schuldausschluss zuzulassen. Die Bank kann sich dann gegen das Unrecht wehren und die Polizei den unrechtmässigen Zustand beseitigen, zugleich sind die AktivistInnen freizusprechen. Als Tennis spielende WhistleblowerInnen für das Weltklima trifft sie kein Schuldvorwurf.

96 Urteil des EGMR 28495/06 und 28516/06 vom 17. Mai 2011 (Akgöl und Göl gegen die Türkei), \$43. («The Court ... considers that a peaceful demonstration should not, in principle, be made subject to the threat of a penal sanction.»). Zu Recht hebt FONJALLAZ, Avis minoritaire (Fn.10) hervor, dass dort, wo friedliche und gewaltfreie Demonstrationen legitime Drittinteressen («les activités licites d'autrui» [wie in casu das Hausrecht der CS]) beeinträchtigen, die Zulässigkeit von Sanktionen in einer Gesamtabwägung zu beurteilen ist.

97 Zur Ableitung der Wahrung berechtigter Interessen aus dem Notstand per analogiam: BGE 94 IV 68 E. 2; oben bei Fn. 59. 